\title{
L'éthique des professionnels de la communication interne : éthos, praxis et artéfacts
}

\author{
Charline Gorez, Junior Officer, \\ Fédération Belge du Secteur Financier (Febelfin), \\ charline.gorez@gmail.com
}




\title{
Résumé
}

On ne compte plus de nos jours les publications et les études consacrées à la communication interne. Néanmoins, un domaine peine toujours à s'imposer dans les discours : l'éthique. Pourtant, la professionnalisation du métier de communicateur interne constitue un terrain fécond pour investiguer plus en profondeur la discipline. Dès lors, une question a été posée : dans quelle mesure l'éthique est importante pour ce pan de professionnels ? La concrétisation de cette problématique générale a été sous-tendue par trois questions plus spécifiques : comment l'éthique est perçue par les communicateurs internes ? Comment est-elle pratiquée par ces derniers ? Est-elle soumise à des tensions? C'est au travers d'un questionnaire et des entretiens sous la forme de récits de pratiques que les réponses aux questionnements portés par cette recherche ont été obtenues. Par la suite, l'ensemble de ce socle de données a été analysé à la lumière de trois notions : l'éthos (les valeurs), la praxis (les pratiques) et les artéfacts (les objets).

Mots-clés : éthique ; communication interne ; déontologie ; professionnalisation ; identité.

\begin{abstract}
At present, the number of publications and studies dealing with internal communication is quite impressive. However, much less attention goes to the subject of ethics. Now that internal communication is increasingly becoming a matter entrusted to professionals, one should take a closer look at ethical conduct. So, the question is to what extent ethics may be important for those professionals? Any concrete approach to this general issue rests upon the following three specific questions: how do people in charge of internal communication perceive ethical matters? How do they apply ethical principles? Is this a matter subject to tensions? In order to find the answer to those questions, the author has relied on a questionnaire and on a number of interviews with people giving examples taken from practice. The whole of the data has then be put through an analysis based on the notion of "ethos" (values), "praxis" (application) and "artefacts" (tools).

Keywords: ethics; internal communication; code of conduct; professionalization; professional identity
\end{abstract}




\section{Introduction}

Discipline intellectuelle, l'éthique constitue une source de réflexion permanente. Le foisonnement de productions et de pratiques liées à la sphère en est d'ailleurs le parfait exemple.

Dans la richesse de tous ces questionnements, un domaine semble être à la traîne : la communication interne. En effet, « one of the lesser studied aspects of internal communication is the consideration of ethics » (Ruck, 2014, p.52). Pourtant, le contexte de professionnalisation que connait le métier constitue un réservoir fécond de questionnements sur l'identité du communicateur interne.

Faire l'économie de la réflexion sur l'éthique ne semble toutefois pas une perspective heureuse si l'on prend le parti de dire qu'elle garde ouverte la question des objectifs de la pratique professionnelle. Brigitte Bouquet le soulignait, « l'éthique individuelle et professionnelle doit sans cesse être réactivée, réinstituée, au travers de nombreux questionnements et de nombreuses réflexions concrètes » (Bouquet, 2003, p.85).

C'est cet environnement qui nous a conséquemment poussé à porter notre attention sur l'éthique professionnelle du communicateur interne. Une éthique qui, il ne faut pas l'oublier, s'inscrit dans un contexte organisationnel.

Dès lors, nous nous sommes spécifiquement demandé dans quelle mesure l'éthique est importante pour ce pan de professionnels ? La concrétisation de cette problématique générale est par ailleurs sous-tendue par trois questions de recherche : comment l'éthique est perçue par les communicateurs internes ? Comment est-elle pratiquée par ces derniers ? Est-elle soumise à des tensions ? De là, nous avons formulé l'hypothèse suivante : il existe un décalage entre les discours et les pratiques. Cette hypothèse a été élaborée en considérant que l'analyse éthique devrait être vue sous l'angle de la relation dialectique entre les valeurs et les pratiques.

\section{Méthodologie}

Pour tenter de trouver des réponses à ces questionnements ${ }^{1}$, un premier outil de récolte de données a été la mise en œuvre d'un questionnaire pour l'Association Belge de la Communication Interne (ABCI). Celle-ci se définit comme une association qui épaule, en formant et informant, les professionnels de la communication interne. ${ }^{2}$ Il faut savoir que le questionnaire n'a pas été conçu spécifiquement pour cette étude

\footnotetext{
${ }^{1}$ Cette recherche fût réalisée dans le cadre du mémoire en vue de l'obtention du diplôme de Master en Information et Communication de l'Université Catholique de Louvain, année académique 2013 2014.

${ }^{2}$ Voir le site internet : http://www.abci.org/
} 
mais s'inscrivait dans une recherche plus large. En effet, celle-ci consistait en une enquête de satisfaction auprès des membres de l'ABCI. 211 questionnaires ont ainsi été envoyés par emails à l'ensemble des membres de l'association. 70 professionnels de la communication interne ont répondu représentant ainsi un taux de réponse d'environ 33\%. L'envoi s'est effectué du 24 février au 24 mars 2014.

Trois questions du questionnaire ont été retenues pour cette contribution. Celles-ci touchaient à notre sujet en abordant l'éthique professionnelle :

- Quelle importance attachez-vous en tant que membre aux objectifs de l'ABCI : l'établissement d'un code/d'une charte déontologique ?

- Connaissez-vous le code de déontologie de l'ABCI ? ${ }^{3}$

- L'utilisez-vous?

La suite de la méthodologie retenue repose sur 11 entretiens qualitatifs d'environ une heure de communicants internes. Nous avons tenté dans cette recherche de privilégier les récits de pratiques. Ces derniers constituent une forme particulière d'entretien : l'entretien narratif. Les entrevues n'ont pas été menées via une grille d'entretien stricto sensu même si leur passation comportait des questions générales automatiquement traitées. Par exemple, nous avons demandé à chaque communicateur de nous décrire une journée de travail, leurs pratiques quotidiennes et les types de productions qu'ils effectuent. Il était voulu de ne pas aborder la question éthique directement. Cette dernière était toutefois évoquée par la suite lorsque nous demandions en quoi considéraient-ils ces pratiques et productions comme éthiques. Nous avons pris les premiers contacts grâce à l'ABCI. C'est aussi à la suite de recommandations que nous avons été en relation avec d'autres professionnels. C'est pour cette raison que nous nous retrouvons avec des membres et des non-membres de l'association.

En ce qui concerne l'aspect analytique, celui-ci a été conduit à la lumière d'une théorie empruntée à Baillargeon, Brulois, Coyette, David, Lambotte, et Lépine (2013). L'article de base se nomme Figures et dynamiques de la professionnalisation des communicateurs et se retrouve dans le premier Cahier du Resiproc appelé Dynamiques, tensions et vecteurs de professionnalisation des communicateurs. Dans ce travail, les chercheurs ont analysé leurs entrevues à l'aune de trois formes que prend la figure du professionnel en communication : les valeurs (éthos), les actions (praxis) et les objets (artéfacts) (Baillargeon et al., 2013). L'éthos peut être ainsi vu comme une posture réflexive dans les discours, comme un idéal type de la figure. Pour faire le lien avec la recherche du Resiproc sur la figure du professionnel de la communication, l'éthos représenterait « une attitude évaluative [...], en regard de la

\footnotetext{
${ }^{3}$ Le 23 mars 2017, l'ABCI a adopté un nouveau code de déontologie. Notre recherche se base sur l'ancien code établi.
} 
conception que le professionnel se fait de la réalité de sa profession » (Geertz cité dans Baillargeon et al., 2013, p.16). Concernant la praxis, celle-ci renvoie simplement aux actions entreprises pour légitimer une figure. Elle « englobe les gestes, les interventions, les prises de position [...]» (Baillargeon et al., 2013, p.17). Enfin, les artéfacts sont « des objets qui agissent comme médiateurs, pour attester de la présence d'une figure $[\ldots] \gg$ (Ibidem).

Nous avons, quant à nous, identifié les discours, les pratiques et productions qui tendent à dévoiler une certaine figure de l'éthique. La suite de la méthodologie des auteurs dont nous nous inspirons consiste à mettre en rapport dialogique les différentes données classées selon les trois catégories décrites. Cette confrontation peut être menée à bien grâce à une approche matricielle. Nous avons, par conséquent, classé les données de chaque entretien dans des matrices afin de mettre en exergue les points de tension et/ou de convergence. L'analyse viendra de la lecture des matrices.

Au final, deux volets d'analyse seront abordés. En effet, l'un s'évertuera à se demander si les communicateurs internes mobilisent de manière explicite la question éthique dans la pratique et la production d'artéfacts. Ou si, à l'inverse, ils ont un propos distinct ou peu articulé entre d'un côté leur discours sur l'éthique et de l'autre leurs pratiques et leur production d'artéfacts. L'autre niveau cherchera à analyser si sur base d'un discours et d'un éthos éthique, les praticiens ont la volonté de penser, de dépeindre des procédures ou des processus (praxis) réalisant le discours éthique ou s'ils veulent produire des artéfacts codes, chartes ou autres réalisant l'éthique.

\section{Envisager l'éthique dans le contexte de professionnalisation}

L'ensemble de ces questionnements s'ancre dans un contexte bien précis. En effet, la communication interne est considérée comme marquée par le sceau de la professionnalisation (Baillargeon et al., 2013). C'est d'ailleurs dans ce contexte particulier que l'émergence de la réflexion sur l'éthique semble prendre son sens. Ainsi, on peut mettre en exergue le rôle de l'éthique et surtout de son aspect formalisé dans sa dimension de confirmation identitaire. De fait, l'objet éthique intervient chez de nombreux auteurs (de La Broise, Walter, Grevisse...) dans la considération d'une profession. Même si les concepts d'éthique et de déontologie sont différents, ils peuvent être nécessairement mis en tension. La déontologie semble importante pour la professionnalisation mais elle questionne et renvoie premièrement à l'éthique professionnelle.

Afin de mieux saisir le propos, une clarification conceptuelle des notions d'éthique et de déontologie semble nécessaire. L'éthique serait ainsi considérée comme une introspection préliminaire aux actions et aux choix posés. Elle impliquerait une interrogation permanente sur les principes et supposerait une réflexion d'ordre critique (Mercier, 2004). L'éthique serait aussi contingente c'est-à-dire que d'autres 
individus, dans la même situation, peuvent faire un autre choix. Elle serait relative, c'est-à-dire propre à un individu, à ses préférences à un moment donné et enfin, elle porterait sur des fins (Scieur, 2013). La déontologie fait, quant à elle, référence à l'ensemble de règles dont se dote une profession «au travers d'une organisation professionnelle qui devient l'instance d'élaboration, de mise en œuvre, de surveillance et d'application de ces règles » (Mercier, 2004, p. 5). Comme nous l'avons mentionné, les mots «éthique » et «déontologie » peuvent être intrinsèquement reliés :

\begin{abstract}
Il y a entre l'éthique et la déontologie une relation nécessaire, sans quoi la seconde représenterait un système normatif débouchant sur une codification vide de son sens. Effectivement, dans une telle relation, la déontologie apparait comme une série d'ordonnances et d'interdictions se superposant, de l'extérieur, à des activités qui exigeraient pourtant une marge d'autonomie pour qu'elles puissent être qualifiées de professionnelles. Il y aurait là une contradiction qui aurait comme conséquence d'invalider la déontologie dans le champ du professionnalisme [...] La déontologie résulte de l'éthique, mais elle ne doit pas s'y substituer ou tenter d'échapper à sa remise en question qui passe par la réflexion éthique [...] L'éthique professionnelle se situe en quelque sorte en amont et en aval de la déontologie [...] En amont, dans la mesure où elle nourrit cette dernière par des valeurs qui sont susceptibles d'évoluer ; en aval, pour que les valeurs ayant déjà été normalisées ou codifiées soient périodiquement remises en question puis révisées [...] C'est la réflexion éthique qui permet de décristalliser la déontologie [...] (Giroux cité dans Bernier, 2005, pp. 59-60).
\end{abstract}

Pour revenir sur l'articulation entre les concepts d'éthique et de professionnalisation, de La Broise (2013, p.34) nous invite à considérer la formalisation déontologique d'une éthique professionnelle ou d'une morale comme étant des «indices d'une professionnalisation ». Les différents codes et chartes qui suivent la réflexion éthique sont également vus par Walter comme « une pratique sociale à part entière [jouant] le rôle d'indicateurs permettant d'évaluer les transformations éthiques et techniques à l'œuvre dans un champ professionnalisé ou en voie de professionnalisation » (ibidem). De plus, il insiste sur le fait que «pour les professionnels, les codes forment bien une « ressource » construite et mobilisée pour formaliser un modèle ou pour justifier leurs actions, voire leur existence » (de La Broise, 2013, p.39). La composante éthique d'une activité professionnelle participerait ainsi à la délimitation d'univers professionnels (Walter, 2005). Elle constituerait un outil d'édification de l'identité et de la compétence (Walter, 1995). Bernier considère, quant à lui, qu'il n'est pas possible de faire fi des aspects éthiques et déontologiques d'une profession puisque c'est au travers de ces idées que peuvent prendre forme dans la vie quotidienne les libertés et les responsabilités professionnelles. Pour l'auteur, même s'il parle des journalistes dans ce cas-ci, éthique et déontologie sont primordiales dans le processus de légitimation d'une profession (Bernier, 2005, p.25). 
Pour Grevisse (2010, pp. 80-81), qui se réfère également au journalisme, la déontologie est un outil qui régule et évalue la poursuite du bien pour un groupe professionnel. Vecteur d'intégration et de revendication, elle est conçue comme « l'ajustement évolutif de valeurs fondamentales à des situations nouvelles. La déontologie devient alors porteuse d'une identité ouverte et dynamique. » Elle ne peut être réduite à une morale valant pour un groupe particulier et à une époque donnée. Pour lui, ce sont les valeurs éthiques qui sont les socles mais aussi les limites d'un groupe professionnel et de ses identités (Ibidem).

Fitzpatrick et Gauthier (2001), œuvrant dans le domaine des relations publiques, estiment aussi que les principes éthiques sont une étape importante pour légitimer et crédibiliser une profession. Selon ces auteurs, les praticiens ont besoin de bases afin de juger de la justesse des décisions qu'ils prennent tous les jours.

Cependant, ce point de vue n'est pas adopté par certains auteurs qui considèrent que le raisonnement sur l'éthique n'a pas lieu de se dérouler lorsqu'une activité n'est pas professionnalisée (Didier, 2002). Néanmoins, si le débat a le mérite d'exister, il ne doit pas mener à l'absence de considération éthique pour un métier. Didier (2002), dans sa thèse concernant l'éthique des ingénieurs, parle à ce propos d' «éthique sectorielle » qui replace l'examen de l'éthique dans des contextes et situations professionnelles spécifiques. Les dilemmes éthiques ou encore les valeurs partagées ne sont pas les mêmes d'une activité professionnelle à une autre, et par conséquent l'éthique semblerait tout de même jouer un rôle de circonscription et de confirmation identitaire.

L'éthique ne suffit évidemment pas à elle seule pour décrire et prendre la mesure d'une profession. Toutefois, comme l'indique de La Broise (2013, p.48), la réflexion autour d'une notion telle que l'éthique rapportée à l'exercice professionnel « [constitue] des invitations très contemporaines à réfléchir sur le sens et les vertus éventuels de cet exercice ». Aussi, il est moins nécessaire de déterminer si la communication interne relève d'une profession ou d'un métier, que de s'interroger notamment sur la manière dont les communicateurs internes caractérisent leur rôle et leur identité.

\section{Pour une première considération de l'éthique professionnelle des communicateurs internes}

C'est avec la professionnalisation en toile de fond que nous avons d'abord voulu nous intéresser à l'éthique professionnelle des communicateurs internes. Dans cette recherche, celle-ci est balisée par le code de déontologie de l'ABCI.

Comme nous l'avons constaté, le contexte particulier de professionnalisation semble donner sens à l'émergence de la réflexion sur les valeurs structurantes du métier. Pour les auteurs évoqués, tous les domaines d'expertise pourraient ainsi 
renvoyer à des valeurs fondamentales. La légitimité et l'honorabilité d'une profession seraient attestées par l'identification à ces valeurs (Maisonneuve, 2010).

Par conséquent, nous avons voulu nous concentrer premièrement sur cette éthique professionnelle formalisée en voyant comment celle-ci était considérée par les praticiens du domaine de la communication interne. Les résultats du questionnaire semblent offrir un point de départ dans la considération de la perception et de la pratique de l'éthique professionnelle.

Les questions sélectionnées dans le questionnaire permettent d'approcher la perception et la pratique liées spécifiquement au code de déontologie. La réflexion sur l'importance de l'établissement d'un code ou d'une charte de déontologie pour la communication interne semble se rapprocher d'un aspect plus discursif et prospectif de l'éthique professionnelle. En effet, cette question s'approcherait davantage de la notion d'éthos que de praxis ou d'artefact. Dans cette recherche, il apparait que l'instauration d'un document déontologique est importante pour la plupart des communicateurs internes interrogés (rappelons-le, 70 au total). En effet, ils sont un peu plus de 50\% à considérer cela «plutôt important » et près de $20 \%$ à juger cela «très important».

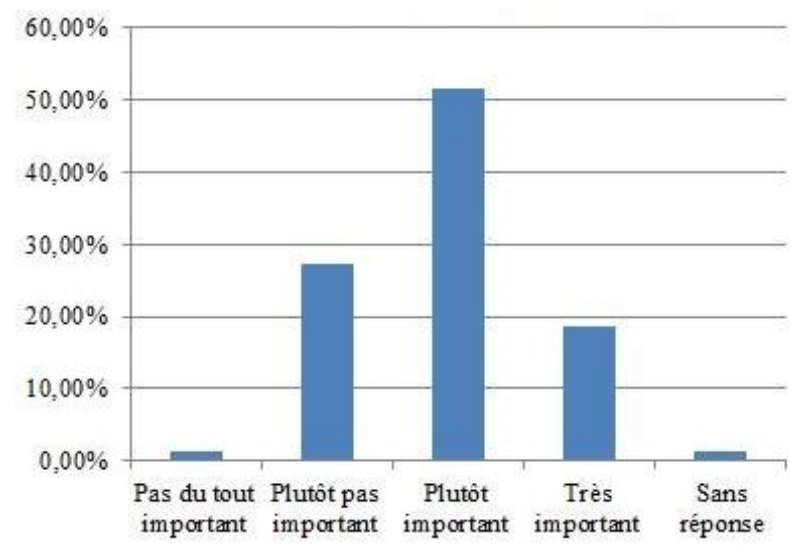

\section{FIGURE 1}

\section{Quelle importance accordez-vous à l'établissement d'un code/d'une charte de déontologie?}

Les deux dernières questions, quant à elles, semblent se distinguer du pôle prospectif pour aborder les faits. Effectivement, demander s'ils connaissent le code déontologie et surtout s'ils l'utilisent interroge davantage les pratiques. 
39 L'éthique des professionnels de la communication interne

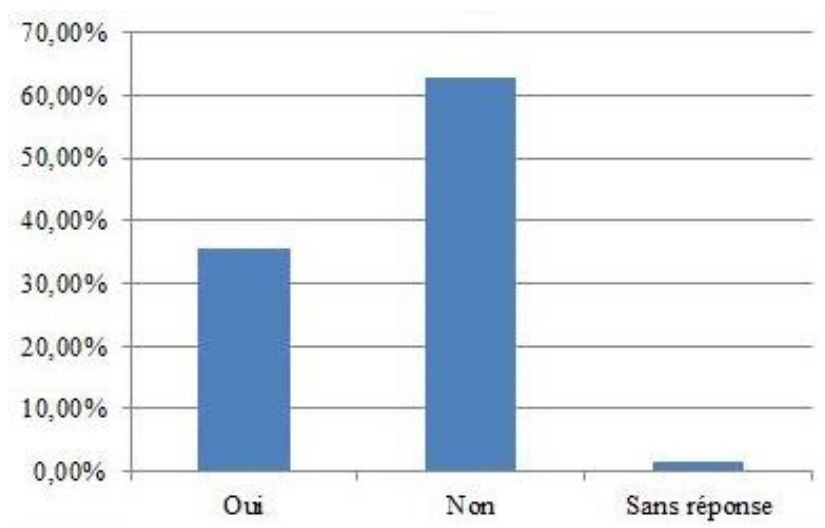

\section{FIGURE 2}

Connaissez-vous le code de déontologie de l'ABCI ?

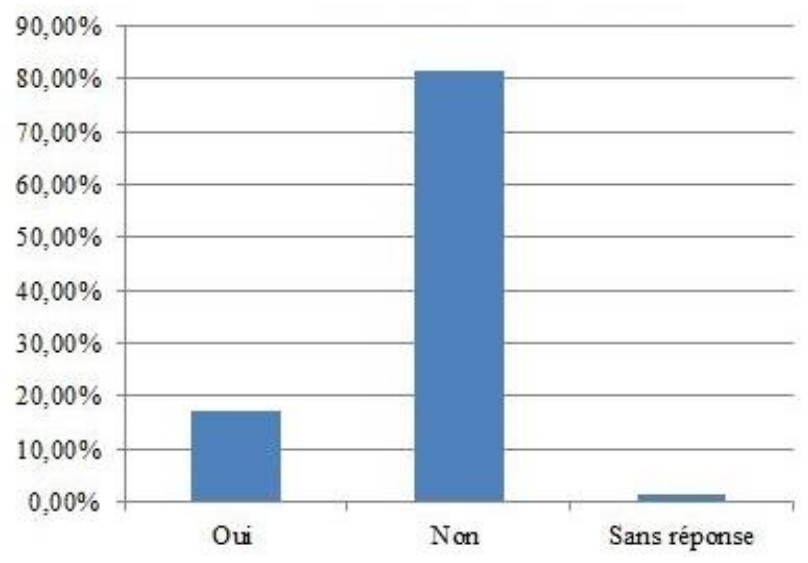

\section{FIGURE 3}

\section{Utilisez-vous le code de déontologie de l'ABCI ?}

Nous le voyons, une différence apparait entre d'un côté l'importance que les membres témoignent pour le code, et de l'autre, la connaissance qu'ils en ont. En outre, même s'ils le connaissent (36\%), ils sont environ la moitié à l'utiliser (17\%).

Ce constat met en relief une désarticulation entre le discours sur l'importance du code de déontologie et son utilisation dans la pratique effective. Le présent 
questionnement révèle ainsi le fait que le code de déontologie de l'ABCI n'est pas un référent éthique pour les communicateurs internes. A un niveau plus large, nous pourrions nous demander si, au final, ce n'est pas toute l'éthique professionnelle des communicateurs internes qui est ignorée dans la pratique ?

Cette remarque n'est pas sans rappeler certaines observations de théoriciens qui mettent en évidence le fait qu'un praticien décidera d'être éthique ou non indépendamment de l'existence d'un code éthique professionnel.

\begin{abstract}
Most of ethics for communication-related associations are filled with meaningless rhetoric, do not accomplish much and are not taken seriously by most of the people who work in. These codes might be able to make ethical behavior less likely because of awareness. With or without professional codes of conduct, most who practice communication will choose to be ethical because they want to be, not because they have to be. (Wright, 2006, pp. 183-184).
\end{abstract}

Cette limite des artéfacts déontologiques a également été pointée par le Resiproc. En effet, selon les auteurs, les documents de type déontologique ne semblent pas utilisés dans les pratiques des professionnels (Baillargeon \& al., 2013).

In casu, nous pourrions alors faire l'hypothèse que la praxis éthique des communicateurs internes est une pratique de type autorégulatoire et ce, sans faire fi du souci déontologique qui semble animer le métier. La perspective autorégulatoire de l'éthique peut être considérée comme «un questionnement sur les valeurs, les normativités et sur notre rapport à l'autre » (Langlois, 2008, para. 10). Elle est un «processus continu d'évaluation du sens de sa pratique et des critères éthiques qui donnent ce sens et le recréent aux détours de situations inédites » (Patenaude \& Chaplin cités dans Langlois, 2008, para. 10). L'autre perspective, hétérorégulatoire cette fois, renvoie à une éthique qui « mise sur des principes [...] qui encadrent et guident les décisions » (Langlois, 2008, para. 9). Cela signifie que les professionnels vont se référer, par exemple, à un code de déontologie qui propose « des éléments qui différencient ce qu'est une conduite acceptable de ce qu'est une conduite inacceptable dans des situations qui sont propres à leur profession »(Ibidem). Les différentes règles organisationnelles peuvent se retrouver dans cette vision.

\title{
5. Quel éthos éthique dans le contexte organisationnel?
}

Pour continuer l'exploration sur l'éthique des communicateurs internes, nous avons tenté de circonscrire ce que nous pourrions appeler l'éthos, dans le sens où il s'agit de « discours », d'idéal type de l'éthique professionnelle. Les communicateurs interrogés s'appuient essentiellement sur des valeurs afin de décrire cette dernière.

Avant tout, la transparence apparait comme l'une des valeurs les plus citées. « Nous voulons être sûrs que les collaborateurs soient bien informés de ce qui se passe dans 
l'entreprise, de la façon la plus transparente possible en communiquant de manière efficace et positive mais honnête » (anonyme - organisation privée).

La transparence semble depuis longtemps nourrir un discours sur l'éthique de la communication. Celle-ci peut être vue tantôt sous des aspects positifs tantôt sous des aspects négatifs. Ainsi, comme le soulignait Boneu (cité dans Libaert, 2003, p.76), « un devoir de cohérence et de transparence s'impose si l'entreprise veut faire de ses salariés de réels partenaires. » En outre, elle ne se résumerait pas au fait de rendre l'information disponible mais impliquerait aussi une «active participation in acquiring, distributing and creating knowledge » (Gower, 2006, p.96). En ce sens, elle peut être vue comme un processus (Ibidem). La notion de transparence semble aussi avoir évolué au fil des années pour la fonction communication interne en se faisant plus présente.

\begin{abstract}
Avant les discours étaient beaucoup plus formatés et quelque part, on infantilisait un peu les collaborateurs entre ce que la direction nous disait et le message qui ressortait aux collaborateurs, il y avait beaucoup de couches de réflexion, « on ne peut pas dire ça » [...] Avant, on ne disait pas ça. Tel projet, tout était toujours beau, le projet se passait super bien alors que tout le monde savait pertinemment que c'était bof... C'est ça la transparence en fait. On ose dire quand ça ne va pas. (anonyme - organisation privée).
\end{abstract}

Si l'on s'attarde quelque peu sur les points négatifs, la transparence pourrait être considérée comme une «notion passe-partout à fort contenu idéologique » qui repose sur des fondements erronés (Libaert, 2003, p.135). L'un d'entre eux, touchant directement notre sujet, serait de considérer la transparence comme la vérité.

L'origine du déplacement sémantique s'explique aisément. Puisque l'opacité étant assimilée à l'acte délibéré de dissimulation [...] donc à la manipulation et in fine au mensonge, son antonyme fut assimilé à la recherche de la vérité puis à la vérité elle-même. La transparence serait le signe de l'authenticité [...] (ibidem, p. 136).

Cependant, c'est le rapport entre la transparence et la confidentialité qui semble constituer un premier point de tension pour les communicateurs internes. Evidemment, l'éthique professionnelle ne doit pas faire fi de l'exigence et du respect de la confidentialité. Là n'est pas le propos. Mais un certain nombre d'entre eux pointent la difficulté, éthiquement parlant, de ne pouvoir rien dire quant à l'évolution de certains dossiers. La valeur transparence et la valeur confidentialité semblent alors se confronter dans la pratique, à certain moment, créant une contrainte pour le praticien.

Il y a des accords de confidentialité, il est certain qu'on ne peut pas tout dire au nom de la transparence, du moment qu'on l'explique également [...] On est parfois au courant de choses avant de pouvoir les transmettre [...] Mais le personnel se pose des questions. Et accuse «oui on ne sait pas ça » et vous savez qu'il y a 
quelque chose qui se prépare. Ça vous brûle les lèvres car on a envie de dire "arrête de critiquer ». Là, il y a effectivement une question d'éthique professionnelle qui joue car on est au courant mais on ne peut pas transmettre. Je dois trouver autre chose pour le contrer plutôt que de franchir la ligne de confidentialité. Voilà, ça je pense que dans beaucoup d'entreprises ça sera le cas. Là où la communication interne est mise très tôt au courant pour pouvoir se préparer, elle a un devoir de confidentialité, de réserve à un moment donné. On ne peut pas transmettre et puis d'un autre côté, il y a vraiment des fois où vous avez envie de leur dire quelque chose mais on doit rester à notre place. (anonymeorganisation privée).

La loi Renault ${ }^{4}$ a également été pointée comme une limite de la transparence. Même si l'on parle de respect de la loi, la contrainte sur le partage d'informations semble toutefois constituer un point de tension éthique pour les praticiens.

\begin{abstract}
A la création de $* * *$, on a dû passer par une phase un peu difficile qui était une info consultation. C'est quelque chose qui n'est jamais très agréable car il s'agit de la loi Renault en Belgique où il faut expliquer quelle est la nature de la réorganisation et quels sont les impacts. Et les impacts, c'est toujours la même chose, c'est la diminution de personnes. Et donc, pendant trois mois, on a été obligé de se taire vis-à-vis de nos employés parce que pendant la négociation avec les syndicats, rien ne pouvait filtrer ... Je peux vous dire que ça ne rigolait pas beaucoup l'année dernière. Les managers étaient fort sollicités et moi je l'étais en tant que manager par des gens qui vous disent «et demain je deviens quoi »? Derrière, il y a des lois, des contraintes et donc à ce moment-là, on est obligé de répondre : je comprends ta situation, tu connais la situation et la loi qui va avec et tu sais que je ne peux rien dire. Quand je pourrai donner les informations, je les donnerai. (anonyme-organisation privée).
\end{abstract}

La transparence est promue par la majorité des communicateurs internes mais celleci doit faire place à la confidentialité en fonction des cas. Cette dernière fait partie de la vie organisationnelle mais peut engendrer des questionnements et des difficultés pour le professionnel.

Le «parler vrai » se retrouve également dans de nombreux écrits consacrés à la communication (Maisonneuve, 2010). Pour certains, la vérité est par ailleurs « la mesure étalon » (Maisonneuve, 2010, p. 379). Ainsi, on retrouve un « double enjeu éthique et épistémologique du discours vrai : la confiance dans l'interlocuteur et la crédibilité des messages. Seule l'information correcte et la plus impartiale possible permet le questionnement et augmente le savoir ; elle seule donne les clés pour juger, pour comprendre, pour agir et s'orienter » (ibidem). Effectivement, ces notions de

\footnotetext{
${ }^{4}$ En bref, la loi Renault en Belgique impose à une entreprise qui annonce ou prévoit un licenciement collectif de mettre en place un processus d'échange d'informations et de concertation entre les interlocuteurs sociaux (motifs du licenciement, etc.). Un régime de sanctions est prévu pour les entreprises ne respectant pas les procédures.
} 
confiance et de crédibilité mises en lien avec l'honnêteté ont été évoquées par certains des communicateurs internes interrogés. Pour ces derniers, cela n'aurait pas de sens pour un spécialiste en communication de mentir.

\begin{abstract}
En tout cas il y a un principe qui est central, c'est qu'on ne ment pas. On ne m'a jamais demandé de mentir dans les communications et je ne serais pas prêt à le faire de toute façon ... Tôt ou tard vous perdez les gens ... Car vous pouvez mentir un mois, deux mois, un an, deux ans mais au final vous perdez toute crédibilité. Et pour un communiquant particulièrement et c'est vrai pour un dirigeant d'entreprise, pour un superviseur qui va gérer des personnes, si vous êtes rattrapés, si on vous met en face de vous là vous avez menti, comment faire confiance après ? (anonyme-organisation privée).
\end{abstract}

Un troisième point assez prégnant dans le discours des praticiens a été de lier la notion d'éthique avec l'objectif principal de la communication interne : la gestion optimale de la demande et de l'offre d'informations dans l'ensemble de l'organisation (d'Almeida \& Libaert, 2010). De son rôle d'intermédiaire, le communicateur interne doit pouvoir trouver ce qui fait sens entre le haut et le bas ou entre les différents services.

Être des porte-voix des collaborateurs qui est le public le plus important. Essayer de ne pas uniquement être utile à la direction mais aussi faire du bottom-up ... Je pense qu'on a tous cette préoccupation du respect du point de vue des collaborateurs, le souci que les gens soient informés ... (anonyme-organisation privée).

Au final, ces trois valeurs les plus citées, de même que les autres évoquées au cours des entretiens, sont liées aux valeurs promues dans l'organisation. Nous verrons plus loin que la profession n'est que peu, voire pas du tout, citée comme référent éthique. Nous nous retrouvons par conséquent avec une perception de l'éthique liée à l'organisation, éthique que les communicateurs internes ont tout de même parfois du mal à délimiter. Effectivement, ils ont été plusieurs à se questionner sur ce que recouvre ce terme. "Ce n'est pas une question que, franchement, on se pose très souvent $\gg$ (anonyme-organisation privée).

Est-ce que la critique vient du fait que la personne ne sache pas définir la notion ou si elle doit être faite à un niveau supérieur ? Néanmoins, la perception et la définition de l'éthique semblent pouvoir être associées avec la manière de concevoir la communication interne et son rôle dans l'organisation.

\title{
6. De l'articulation entre l'éthos et la praxis
}

L'éthique n'a de sens que dans l'action et pour l'action. Si elle peut donner lieu à des spéculations et à des raisonnements théoriques, ce ne peut être qu'en référence 
à l'action dont elle part et dans laquelle elle doit s'inscrire. (Falise \& Régnier, 1992, p.58).

Cette citation rappelle que l'éthique ne peut se limiter à un discours sur une série de valeurs idéales. C'est pourquoi nous allons nous intéresser à l'aspect pratique en nous demandant si celui-ci est encadré par des valeurs.

Un certain souci éthique semble conditionner la manière de fonctionner des praticiens. En effet, il semblerait que l'éthos, la praxis et la production d'artéfacts s'articulent, le premier influençant les deux autres. En d'autres termes, les professionnels de la communication interne se revendiqueraient éthiques.

[L'éthique] ce n'est pas un prétexte de dire : c'est juste un idéal, c'est juste des beaux mots et c'est figé, on l'affiche. C'est un peu comme des valeurs, c'est très beau, pour la décoration du bureau et après dans la réalité on s'en fout, non! (anonyme- agence de communication).

«C'est quelque chose que l'on vit au quotidien sans s'en rendre compte, quand on a déjà été élevé d'une certaine manière, quand on partage des valeurs de son entreprise, ça va se faire naturellement » (anonyme-organisation privée).

«Nos communications sont cohérentes par rapport à ces valeurs éthiques » (anonyme-organisation privée).

Quant aux artéfacts attestant l'éthique, les communicateurs internes interrogés n'en réalisent pas. Seul un praticien a été corédacteur d'une telle production (un code de déontologie).

Si la pratique est encadrée par des valeurs, il faut savoir que ces dernières sont avant tout des valeurs organisationnelles et personnelles.

\subsection{Les valeurs organisationnelles agissent sur la praxis}

Le cadre éthique d'un individu peut lui être amené par trois systèmes de valeurs. En effet, comme l'explique Bivins (2006, p.37):

That framework may be provided by the profession (as a code of professional standards), by the organization for wich a practitionner works (as a corporate code or simply by the corporate culture itself), or by personnal ethical standards. Each of these plays a part in creating the moral ground from wich a true professional makes decisions.

Nous avions fait l'hypothèse dans la partie consacrée au questionnaire que la praxis éthique des communicateurs internes était une pratique de type autorégulatoire. De fait, nous nous étions premièrement intéressées au code de déontologie et avions découvert qu'il ne semblait pas servir de référent pour les praticiens. Cependant, la lecture des entretiens a révélé que la définition du mal ou du bien par ces derniers se 
réaliserait davantage par rapport à l'organisation que par rapport à une éthique de la profession telle que promue par les associations.

Cette perspective fait

En sorte de considérer l'éthique comme intégrant les deux modes régulatoires [...] L'éthique est une forme de questionnement, mais ce dernier s'inscrit dans une structure qui invite les individus à respecter les règles et les principes, pour mieux guider leur conduite au travail ou à trouver les balises nécessaires pour prendre leurs décisions. Ainsi, le mécanisme de prise de décision se rationnalise à partir des critères organisationnels. (Langlois, 2008, para. 10).

L'organisation et son climat éthique pèseraient ainsi sur le comportement éthique des salariés. De manière générale, les individus sont pétris par les valeurs organisationnelles en étant fortement influencés par la culture de l'organisation.

Ce système informel de valeurs est également formalisé par différents codes, chartes... qui offrent aux membres «un cadre de référence pour l'action» et une identité commune (Mercier, 2000, p.109).

Ce ne sont pas des valeurs théoriques très développées mais ce sont des valeurs finalement de base et qui peuvent permettre de diriger l'ensemble des comportements, des communications, des processus de reconnaissance que l'on peut avoir. Et pour ça, le code de conduite de $* * *$ est finalement assez bien fait. (anonyme-organisation privée).

En outre, cette prédominance des valeurs organisationnelles dans la pratique éthique des communicateurs internes pourrait s'expliquer de par le rôle même de la communication interne par rapport à ces valeurs. De fait,

La branche d'activité la plus déterminante pour la promotion de l'éthique à l'intérieur de l'organisation est la communication interne [...] Ainsi, grâce à la communication interne sont diffusées auprès des employés les valeurs d'entreprise de l'institution pour que les mêmes employés diffusent ces valeurs auprès des différents groupes d'intérêts [...] (Medina-Aguerrebere, 2013, para.12).

Les valeurs de l'organisation seraient leurs références car ce sont eux qui, pour la plupart, sont chargés de les transmettre et de les communiquer. Cependant, l'éthique est par définition individuelle. Par conséquent, il ne faut pas oublier les valeurs personnelles dans la considération de la praxis.

L'éthique organisationnelle ne peut s'inscrire dans les pratiques que si elle fait sens pour les acteurs. C'est-à-dire si elle est cohérente avec les significations mentales que les personnes construisent par rapport à eux-mêmes, à leurs engagements et à leur activité professionnelle. (Saielli, 2001, para. 44). 
Ainsi, les valeurs organisationnelles semblent devoir s'accorder avec les valeurs personnelles de l'individu. La sensibilité éthique d'une personne, de même que son développement, semble donc être la résultante d'un alliage entre ces deux composantes. «Si je travaille depuis si longtemps dans cette entreprise, parce que ça va faire 18 ans en octobre, c'est que quelque part, je me reconnais dans ses valeurs » (anonyme-organisation privée).

C'est lorsque l'alignement n'est pas rencontré que le communicateur interne peut faire face à des dilemmes ou à des souffrances éthiques. In casu, les professionnels que nous avons interrogés n'ont pas déclaré s'être retrouvés à un moment de leur vie professionnelle confrontés à cela. La plupart sont en effet alignés aux valeurs de leur organisation. Néanmoins, ils ont été plusieurs à mentionner le fait qu'une telle situation d'inadéquation entre les valeurs peut amener à quitter l'organisation.

Ne pas rencontrer de dilemmes ne signifie pas qu'une tension ne peut toutefois pas apparaitre entre les valeurs organisationnelles et les valeurs personnelles. En effet, le communicateur interne peut être amené à devoir accepter certaines valeurs de l'organisation. Il exercerait ainsi un glissement en faveur des valeurs organisationnelles, ne fut-ce que pour la préservation de l'emploi.

Il faut s'adapter à la philosophie de l'entreprise, on fait avec ... On a beau avoir sa propre éthique et ses propres valeurs, il y a aussi celle de l'entreprise quelle qu'elle soit ...Je crois qu'il y a l'éthique propre, personnelle et déontologique avec tout ce qu'on nous a appris dans le métier et puis, il y a l'éthique de l'entreprise sur laquelle on est forcé de se calquer, qu'on y croit ou qu'on n'y croit pas. (anonymeorganisation publique).

Au-delà même d'une tension entre des valeurs personnelles et des valeurs organisationnelles, il semblerait que la plupart des problèmes touchant à l'éthique seraient davantage liés au décalage entre une valeur organisationnelle et sa traduction.

\subsection{Quid de la profession ?}

Nous l'avons répété, la profession ne semble pas être un cadre de référence éthique pour les communicateurs internes interrogés tant dans le questionnaire que dans les entretiens. Cependant, nous pouvons nous demander pourquoi ?

Il a été vu que l'éthique professionnelle participerait à la délimitation et à la confirmation identitaire d'un univers professionnel. Seulement, la communication interne serait en cours de professionnalisation et amènerait dès lors une certaine imprécision de l'identité. En résulterait alors une incertitude quant à la définition d'une éthique professionnelle?

Quoi qu'il en soit, le communicateur interne comblerait les inexactitudes de son identité en s'assimilant à l'organisation et aux valeurs y afférentes. Les valeurs professionnelles seraient, quant à elles, trop floues voire inconnues pour être 
mobilisées. Sans pour autant nier qu'elles se tissent peut-être en filigrane de manière inconsciente, elles ont des difficultés à s'imposer dans le discours.

En outre, la collecte de données a montré que la mention d'une déontologie se réalise uniquement lorsque la personne a effectué des études en journalisme. Les autres codes connus tels le Code d'Athènes et le Code de Lisbonne ne sont pas non plus mentionnés comme référents. ${ }^{5}$ L'éthique de la communication interne reste ainsi toujours dans une certaine opacité et l'organisation semble demeurer prioritaire dans la hiérarchisation des valeurs. La formation reçue semblerait quand même jouer un rôle dans la prise de conscience d'une éthique professionnelle.

\begin{abstract}
La théorie est très claire et les règles déontologiques en journalisme sont très claires. Et là c'est l'exemple type qu'il y a des règles déontologiques et qu'on voit dans la pratique qu'elles sont interprétées de manières très diverses selon le média pour lequel on travaille. Je pense qu'en communication interne... Moi je n'ai pas connaissance de règles éthiques. Je pense que chacun travaille aussi un peu en fonction de sa population. Je pense qu'en communication interne, notre boulot dépend de l'entreprise dans laquelle on travaille, du secteur, de la culture d'entreprise et des populations ... L'éthique, c'est quelque chose de très vague encore en communication interne. (anonyme - organisation privée).

Déjà, ce ne sont pas mes études à la base. Donc je ne sais pas ce qu'on apprend, si quelqu'un fait de la com à l'unif ou même en graduat, enfin en baccalauréat, je ne sais pas du tout si on apprend une certaine éthique. (anonyme - organisation privée).
\end{abstract}

Enfin, certains pointent le fait que l'éthique professionnelle n'apporterait pas de plus-value à celle de l'organisation, surtout si la vie au sein de celle-ci s'avère positive.

Si l'organisation est la référence éthique, celle-ci s'incarne notamment dans les individus qui la composent. Eux-mêmes, de par leur perception et leur pratique, incitent ou non aux comportements éthiques.

\title{
6.3. La direction et le management, « boosteurs » de la pratique éthique?
}

Le climat éthique de l'organisation est composé de personnes qui contribuent à sa création (Mercier, 2004). Dans cet ordre d'idées, Posner et Schmidt (cités dans Mercier, 2004) ont montré que les supérieurs et les collègues exercent une influence considérable dans les agissements éthiques.

\footnotetext{
${ }^{5}$ Ils traitent davantage du domaine des relations publiques. Le Code d'Athènes est nommé plus explicitement « Code d'Ethique International des praticiens de relations publiques » et le Code de Lisbonne «Code Européen de Déontologie Professionnelle des Relations Publiques ».
} 
Ainsi, la personnalité et la sensibilité éthique de ces derniers joueraient un rôle essentiel pour l'établissement d'un climat éthique efficace. Il s'agit d'un premier élément permettant le développement d'une praxis éthique de la part des communicateurs internes. Mais cela ne serait pas tout. De fait, dans le cas de la communication interne, un lien peut être réalisé entre la légitimité de la fonction et le comportement éthique. Dans ce cas de figure, il semble plus facile de défendre un comportement éthique lorsque l'on atteint une certaine légitimité et reconnaissance.

La question de la direction et de sa conception de la communication interne est revenue plusieurs fois dans les entretiens lorsque les praticiens abordaient l'éthique. Le fait d'être légitime permettrait en effet de mener à bien ses missions et rôles et, par là, de pouvoir mettre en pratique une série de valeurs. Cette légitimité impulserait un développement d'une praxis basée sur un éthos. Elle les lierait.

Dans l'ensemble du comité de direction, tout le monde reconnait l'importance de la communication interne. Donc c'est vrai que j'ai la chance d'avoir des gens qui supportent parce qu'ils sont convaincus des bienfaits de la com interne sur la motivation, sur tout ce qu'on peut imaginer ... Il y a 10 ans de cela, ce n'était pas le cas, c'était un secrétariat amélioré tandis que là, maintenant depuis 5 ans, vraiment c'est quelque chose qui est vu comme essentiel. (anonyme- organisation privée)

\subsection{Les communicateurs internes, influenceurs de la pratique éthique}

Si la direction et le management ont un effet sur le comportement éthique, il faut savoir que cette influence n'est pas unilatérale. Le communicateur peut, à son tour, influencer la pratique éthique.

L'un des objectifs de la fonction étudiée consiste bien à aider et conseiller le management dans son rôle d'information, d'écoute et de dialogue. Le fait de conseiller peut amener à proposer un point de vue plus éthique. Le communicateur interne ne se percevrait plus comme une «danseuse» de la direction (une des critiques les plus réalisées à leur encontre) mais comme un acteur promouvant des attitudes éthiques dans l'organisation.

Sincèrement, je dirais que le côté de danseuse de la direction générale, c'était peutêtre au début, quand j'ai repris la communication interne. Je travaillais pour les deux entreprises et on me disait : tu fais la secrétaire de luxe ... Je n'ai pas l'impression d'obéir au doigt et à l'œil quand on me propose quelque chose. On me demande : qu'en penses-tu ? Après, si je ne suis pas d'accord, on va peut-être me l'imposer quand même mais $\mathrm{j}$ 'ai le droit de donner mon avis. (anonymeorganisation privée). 
Être responsable éthiquement, c'est aussi pour le communicateur interne de conseiller lorsqu'il n'est pas d'accord sur une série d'éléments. Le désaccord qui semble le plus prégnant concerne la transmission d'une information.

En effet, l'éthique peut être soumise à ce type de tensions, même si selon la plupart des communicateurs internes, cela est assez rare. Et c'est d'ailleurs en apportant son opinion que le communicateur interne semble valoriser le lien social et la prise en compte des parties prenantes. Comme ils ont été plusieurs à nous le mentionner, la communication interne est un job d'équilibriste. C'est en privilégiant l'assertivité et la proactivité que le praticien peut préserver cet équilibre afin que se maintienne un lien entre l'éthos et la praxis.

\begin{abstract}
Ma réaction a toujours été en tant que responsable de la communication en disant : voilà, moi j'ai un problème parce que d'un côté, dans notre mission, vision, valeurs, nous prônons un tel message et puis moi, maintenant, je suis obligé de véhiculer un message contraire. Je dis et j'explique que je suis en contradiction. Si les lecteurs, le personnel se rendent compte de cette incohérence, voilà les conséquences. Je dis que moi pour cette raison j'ai un problème. En général, j'ai eu gain de cause ... Mais je crois que le rôle du communicateur, c'est... Je pense qu'il faut faire preuve d'assertivité... Et alors comme aussi parfois dans certaines situations c'est de dire, voilà, moi je ne le fais pas mais c'est vous qui prenez la responsabilité. Alors évidemment, ça fait réfléchir. Donc voilà comment on peut dans certaines situations remédier à une difficulté ... (anonyme-organisation publique).
\end{abstract}

\title{
6.5. La direction et le management : des freins possibles à la pratique éthique?
}

La réflexion sur la légitimité de la fonction revient une nouvelle fois dans notre questionnement. En effet, même si la communication interne se professionnalise de plus en plus, elle est toujours exposée à des obstacles. Comme l'affirme l'étude AFCIInergie (2013),

Le communicant interne est souvent obligé d'apporter encore et toujours la preuve de la valeur ajoutée de sa fonction, ce qui, on l'imagine aisément, peut engendrer une certaine usure. La question - quasi existentielle - pourrait alors être : comment « tenir » et comment ne pas être oublié ?

Une des conséquences serait qu'il doit, dans le même prolongement, apporter des arguments pour que se maintiennent certaines valeurs comme la clarté de l'information ou la transparence. C'est par la défense de sa fonction qu'il peut défendre le personnel et donc prôner une certaine conception de l'éthique. De plus, cette défense semble d'autant plus importante de par le fait que le praticien ne possède 
pas un statut ni une protection. De même, en fonction des contextes, « son accès aux sources d'information n'est pas garanti. Son droit d'informer le personnel est totalement subordonné aux décisions managériales » (Donjean, Hambursin, Lambotte \& Scieur, 2012, p.10).

Pour moi, c'est clair que la com dans notre entreprise n'est pas assez valorisée ... 'C'est que de la com, tout le monde sait faire de la com'. Mon chef précédent me l'a encore dit, 'mais tu n'as pas d'expertise, tu fais de la com'... Ici, je n'ai pas l'impression que les employés sont considérés comme des ambassadeurs. Je suis un peu critique. C'est vrai que c'est un peu dommage cette mentalité. Faut continuer à booster... Pour moi, transformer les employés en ambassadeurs c'est indispensable. Et là ce que j'entends ce sont des collègues qui ne sont pas des ambassadeurs ... Toujours convaincre... Pour véhiculer le même message... ça demande beaucoup d'énergie. Si on n'est pas passionné ça ne va pas. On se brûle. (anonyme - organisation privée).

Ainsi, une certaine résistance au déploiement d'une praxis basée sur plusieurs valeurs peut s'opérer dans le chef du management. D'ailleurs, le fait de considérer la direction et le management comme des freins à la pratique éthique peut se détecter par un changement dans les termes utilisés pour décrire son action. Du verbe « conseiller », on observe un glissement des énoncés vers des notions de défense, de combats, etc. Cela consisterait pour les communicateurs internes en une tension liée à l'éthique.

C'est accepter pour le management, la transparence et la communication claire ... C'est vraiment un combat quotidien de faire prendre conscience à certains top managers qu'ils ne parlent pas à des enfants. Qu'il faut dire les choses, qu'on parle à des adultes ... La capacité de compréhension n'est pas proportionnelle au niveau hiérarchique dans lequel on se trouve. Et il y a parfois, on se dit que ce n'est pas correct de ne pas dire certaines choses ou de ne pas les dire clairement ... C'est très tentant d'utiliser les gens et qu'on a parfois le mauvais rôle en mettant un frein. C'est difficile de trouver un équilibre. (anonyme - organisation privée).

\section{Conclusion}

Les données du questionnaire ont permis de constater que l'éthique professionnelle formalisée est importante aux yeux des praticiens interrogés. Seulement, la considération de la praxis a révélé que la majorité d'entre eux ne l'utilisent pas comme référent dans leur quotidien organisationnel. Dès lors, on assisterait à une désarticulation entre le discours sur l'importance du code de déontologie et son utilisation dans la pratique effective.

L'éthique professionnelle semble difficile à définir. Néanmoins, les réponses obtenues dévoilent que les valeurs prédominantes du communicateur interne, pouvant 
être rattachée à l'éthos, semblent consister en la proposition d'une communication transparente, honnête et respectueuse de la circulation de l'information à tous les niveaux. Le tout, à un niveau plus général, contribuerait à la promotion de la pluralité des opinions et de l'échange d'idées. Ces valeurs seraient ainsi le socle de base de la perception de l'éthique chez les praticiens. Cependant, ces dernières sont intrinsèquement perçues comme étant organisationnelles. Et ce sont ces valeurs qui régiraient la praxis. On assisterait donc à une invalidation de l'hypothèse de départ. En effet, la désarticulation entre l'éthos et la praxis exprimée supra serait contrecarrée, car c'est la norme de l'organisation qui prend le pas sur la norme de la « profession ». Ce constat peut s'expliquer par le fait que le communicateur interne comblerait les inexactitudes de son identité (car le métier est relativement nouveau) en s'assimilant à l'organisation et aux valeurs y afférentes. Les valeurs professionnelles seraient ainsi trop floues pour être mobilisées.

Le lien entre éthos et praxis peut être encouragé ou mis à mal en fonction de divers éléments. Tout d'abord, la direction et le management influenceraient la praxis éthique de par leur personnalité mais aussi de par la manière dont ils envisagent la communication interne. De fait, il semblerait plus facile pour un communicateur interne de défendre un comportement éthique lorsqu'il est reconnu. Par conséquent, la légitimité impulserait le développement d'une praxis liée à un éthos.

Ensuite, le praticien peut inspirer lui-même la praxis éthique. Sujet mais aussi objet d'influence, la responsabilité éthique du communicateur interne semble alors induire la possibilité de développer et de maintenir cette inter influence. Par là même, il participerait à l'émergence d'une communication plus éthique en mettant en place et en animant des processus de dialogue. En cas de désaccord ou non avec ce qui est demandé, le professionnel de la communication interne privilégierait ainsi l'assertivité et la proactivité pour exercer son « job d'équilibriste ». Le but sous-jacent est que se maintienne la relation éthos/praxis. Dans tous les cas, la configuration actuelle de la vie en organisation semble montrer que le communicateur interne a la possibilité de remplir ce rôle particulier. Toutefois, de nouveau concernant la légitimité, la direction et le management peuvent être des freins à l'articulation discours/pratique lorsque la valeur ajoutée du communicateur n'est pas reconnue. Effectivement, une certaine résistance du management apparaitrait quant au déploiement d'une praxis basée sur plusieurs valeurs telles que la clarté, etc. Ce serait alors au praticien d'apporter les arguments de défense de son métier et, par là, les arguments de défense de la relation entre le binôme d'approches. Cette observation consisterait d'ailleurs en la tension principale que puisse connaitre un communicateur interne.

Les différents constats exposés tout au long de cette recherche semblent indiquer que l'éthique relève d'une certaine importance pour les praticiens. Elle sous-tendrait les pratiques même si elle est parfois compliquée à caractériser. Une des raisons est qu'elle ne renvoie pas fondamentalement à une définition univoque. Cependant, ce 
sont les valeurs qui sont au centre de la réflexion éthique. Pour nos professionnels, celles-ci sont envisagées sous un pôle organisationnel en l'absence de véritables valeurs structurantes du métier.

Avant de conclure, nous voulons ajouter que l'éthique est un phénomène en évolution, qui s'actualise dans les pratiques contextualisées. Comme Badiou l'évoquait (cité dans Langlois, 2008, para. 8), "l'éthique n'est pas une catégorie générale, saupoudrée uniformément sur l'ensemble des pratiques, mais elle se nourrit d'un rapport fort au réel, aux faits, aux réalités concrètes et différenciées. » Cette citation soulèverait une limite de la recherche : ne pas avoir pu confronter l'éthos et la praxis par une observation in situ. Une piste serait alors d'observer les éléments mis en avant dans cet article dans différents contextes. En outre, tout en prenant le recul nécessaire, il serait intéressant de réitérer cet exercice de recherche en se basant sur le nouveau code de déontologie de l'ABCI. Celui-ci a été adopté fin mars 2017.

Au final, cet article ne prétend pas épuiser l'étendue de la problématique sur l'éthique et n'a pas la volonté de fermer le débat. En effet, les questions restent multiples et la réflexion sur l'éthique n'est jamais close. Jean Moussé le soulignait parfaitement (cité dans Smets, 2002, p. 87), «l'éthique doit être considérée comme une démarche qui n'a pas de terme. Elle n'en est pas moins urgente. Elle n'est ni un terme, ni un code. C'est un chemin...» 


\section{Bibliographie}

AFCI-Inergie. (2013). Quelle identité professionnelle pour le communicant interne? [PDF en ligne] http://www.inergie.com/accueil/etudes/barometre-inergie-afciquelle-identite-professionnelle-pour-le-communicant-interne/

Baillargeon, D., Brulois, V. Coyette, C., David, M. D., Lambotte, F. \& Lépine, V. (2013). Figures et dynamiques de la professionnalisation des communicateurs. In D. Baillargeon \& M.D. David (Eds.), Cahiers du Resiproc: La professionnalisation des communicateurs : dynamiques, tensions, vecteurs (pp. 12-32). Louvain-la-Neuve : Presses Universitaires de Louvain.

Bernier, M.-F. (2005). Ethique et déontologie du journalisme. Québec : Presses de l’Université Laval.

Bivins, T.H. (2006). Responsability and Accountability. In K. Fitzpatrick \& C. Bronstein, Ethics in public relations: responsible advocacy (pp.19-38). Thousand Oaks : Sage Publications.

Bouquet, B. (2003). Ethique et travail social. Paris : Dunod.

D’Almeida, N. \& Libaert, T. (2010). La communication interne des entreprises. Paris : Dunod.

De La Broise, P. (2013). La lutte pour la reconnaissance ? Codes, chartes, référentiels et autre manifestes de professionnels de la communication. In D. Baillargeon \& M.D. David (Eds.), La professionnalisation des communicateurs : dynamiques, tensions, vecteurs (pp. 33-51). Louvain-la-Neuve: Presses Universitaires de Louvain.

Didier, C. (2002). Ethique et identités professionnelles des ingénieurs. Enquête sur les diplômés des écoles du Nord de la France (Thèse de doctorat). Ecole des Hautes Etudes en Sciences Sociales, Paris. En ligne: http://tel.archivesouvertes.fr/docs/00/77/96/51/PDF/these_CDidier-final.pdf

Donjean, C., Hambursin, C., Lambotte, F. \& Scieur, Ph. (2012). Vers une politique de communication interne socialement responsable en période de restructuration. Etude préliminaire. (Résumé du rapport de recherche). [PDF en ligne] http://www.google.be/url?sa=t\&rct=j\&q=\&esrc=s\&source=web\&cd=2\&ved=0 CCcQFjAB\&url=http\%3A\%2F\%2Fwww.emploi.belgique.be \%2FWorkArea\%2 FDownloadAsset.aspx\%3Fid\%3D37087\&ei=UKPYU4SjG_TX7AbJs4GgBg\& usg=AFQjCNEhBr0iVaZTLuhKNLFdFFVdaYrzfQ\&sig2=fysvNV9o3JYFQ4t 43nRQ9

Falise, M. \& Régnier, J. (1992). Repères pour une éthique d'entreprise. Lille: Centre d'Ethique Contemporaine. 
Fitzpatrick, K., \& Gauthier, C. (2001). Toward a Professional Responsibility Theory of Public Relations Ethics. Journal of Mass Media Ethics, 16(2/3), 193-212.

Gower, K.K. (2006). Truth and Transparency. In K. Fitzpatrick \& C. Bronstein, Ethics in public relations: responsible advocacy (pp. 89-105). Thousand Oaks : Sage Publications.

Grevisse, B. (2010). Déontologie du journalisme. Enjeux éthiques et identités professionnelles. Bruxelles : De Boeck Université.

Langlois, L. (2008). Une éthique à deux vitesses : dangers et répercussions sur l'identité professionnelle. Pyramides, 16(2), 33-52. En ligne http://pyramides.revues.org/210?lang=en

Libaert, T. (2003). La transparence en trompe-l'œil. Paris : Descartes \& Cie.

Maisonneuve, D. (2010). Les relations publiques dans une société en mouvance. Québec : Presses de l'Université du Québec.

Medina - Aguerrebere, P. (2013). Le rôle de la communication dans l'éthique organisationnelle. Communication, 31(2). En ligne http://communication.revues.org/4503

Mercier, S. (2000). La formalisation de l'éthique : un outil stratégique pertinent pour l'entreprise. Finance Contrôle Stratégie, 3(3), 101-123.

Mercier, S. (2004) . L'éthique dans les entreprises. Paris : Editions La Découverte.

Ruck, K. (2010). Exploring internal communication. Harlow: Pearson Education Limited.

Saielli, P. (2001). Analyse critique de l'éthique organisationnelle. Communication et organisation, 20. En ligne http://communicationorganisation.revues.org/2586

Scieur, Ph. (2013). L'éthique de l'information et de la communication. Syllabus, Université Catholique de Louvain, Mons.

Smets, P.-F. (2002). Ethique ou cosmétique. Le retour des valeurs dans un monde paradoxal. Bruxelles : Bruylant.

Walter, J. (2005). Mondes professionnels de la communication et de la transparence. De la codification à la régulation. Anthropologie et communication - MEI, 22, 25-42.

Wright, D.K. (2006). Advocacy across borders. In K. Fitzpatrick \& C. Bronstein, Ethics in public relations: responsible advocacy (pp.171-190). Thousand Oaks : Sage Publications. 